\title{
Successful resection of a slow-growing synchronous pulmonary metastasis from distal cholangiocarcinoma resected 3.5 years after initial surgery: a case report
}

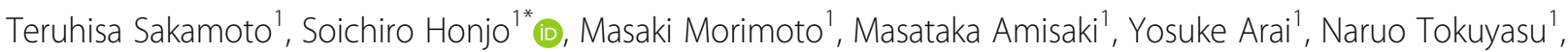
Keigo Ashida ${ }^{1}$, Hiroaki Saito ${ }^{1}$, Kanae Nosaka ${ }^{2}$ and Yoshiyuki Fujiwara ${ }^{1}$

\begin{abstract}
Background: A few reports have described the effectiveness of resection for recurrent cholangiocarcinoma. However, none have described resection of synchronous pulmonary metastasis from distal cholangiocarcinoma. We report the first case, to the best of our knowledge, of a slow-growing synchronous pulmonary metastasis from distal cholangiocarcinoma resected 3.5 years after the initial surgery.

Case presentation: A 67-year-old Japanese man with a diagnosis of distal cholangiocarcinoma was referred to our hospital. Thickening of the distal bile duct and an air-space pattern in the upper lobe of the left lung were detected by preoperative computed tomography. He underwent pancreaticoduodenectomy for the distal cholangiocarcinoma. Follow-up chest computed tomography demonstrated that the air-space pattern in the left lung had gradually enlarged. Thoracoscopic left S6 segmentectomy with lymph node dissection was performed 3.5 years after the initial surgery. Histopathology of the resected specimen revealed a solitary metastasis from distal cholangiocarcinoma with lepidic growth. We diagnosed the patient with a solitary synchronous pulmonary metastasis from distal cholangiocarcinoma.

Conclusions: Surgical resection might offer better long-term survival to patients with synchronous pulmonary metastasis from distant cholangiocarcinoma than nonsurgical treatments. Pulmonary metastasis from distal cholangiocarcinoma may exhibit a lepidic pattern. Therefore, because of the possibility of synchronous pulmonary metastasis, pulmonary resection should be considered for patients with lepidic lesions who have been diagnosed with distal cholangiocarcinoma.
\end{abstract}

Keywords: Synchronous pulmonary metastasis, Distal cholangiocarcinoma, Lepidic growth

\section{Background}

Surgical resection is necessary for curative treatment of distal cholangiocarcinoma. Although systemic chemotherapy is applied to distal cholangiocarcinoma with factors of unresectability such as metastasis to the liver, lung, peritoneum, and distant lymph nodes (LNs), its clinical value remains unsatisfactory $[1,2]$. A few recent reports have described the effectiveness of resection for

\footnotetext{
* Correspondence: honjo@med.tottori-u.ac.jp

${ }^{1}$ Division of Surgical Oncology, Department of Surgery, Faculty of Medicine, Tottori University, 36-1 Nishi-cho, Yonago 683-8504, Japan

Full list of author information is available at the end of the article
}

treatment of recurrent cholangiocarcinoma [3-6]. However, none have described resection of synchronous pulmonary metastasis from distal cholangiocarcinoma. We report the first case, to the best of our knowledge, of a slow-growing synchronous pulmonary metastasis from distal cholangiocarcinoma resected 3.5 years after the initial surgery.

\section{Case presentation}

A 67-year-old Japanese man who had been diagnosed with distal cholangiocarcinoma at a previous hospital was referred to our hospital for surgical treatment. He 

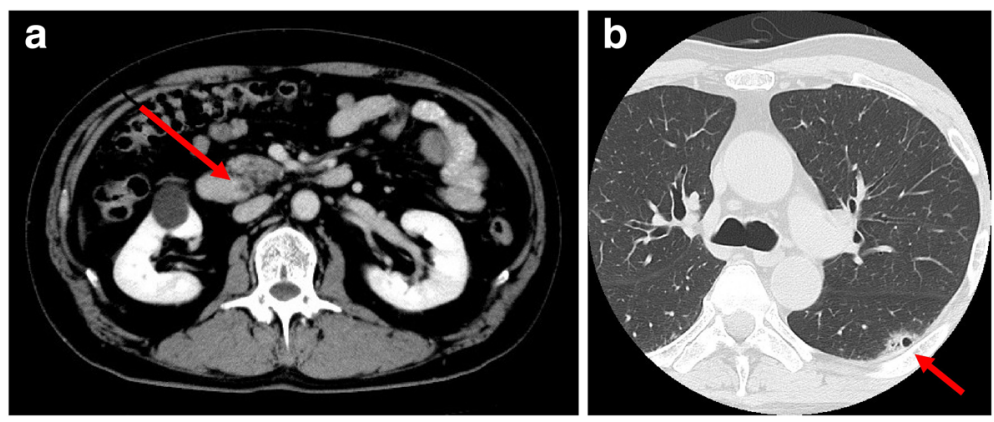

Fig. 1 Computed tomography findings. a Abdominal computed tomography showing thickening of the distal bile duct (arrow). b Chest computed tomography showing an air-space consolidation in the upper lobe of left lung (arrow)
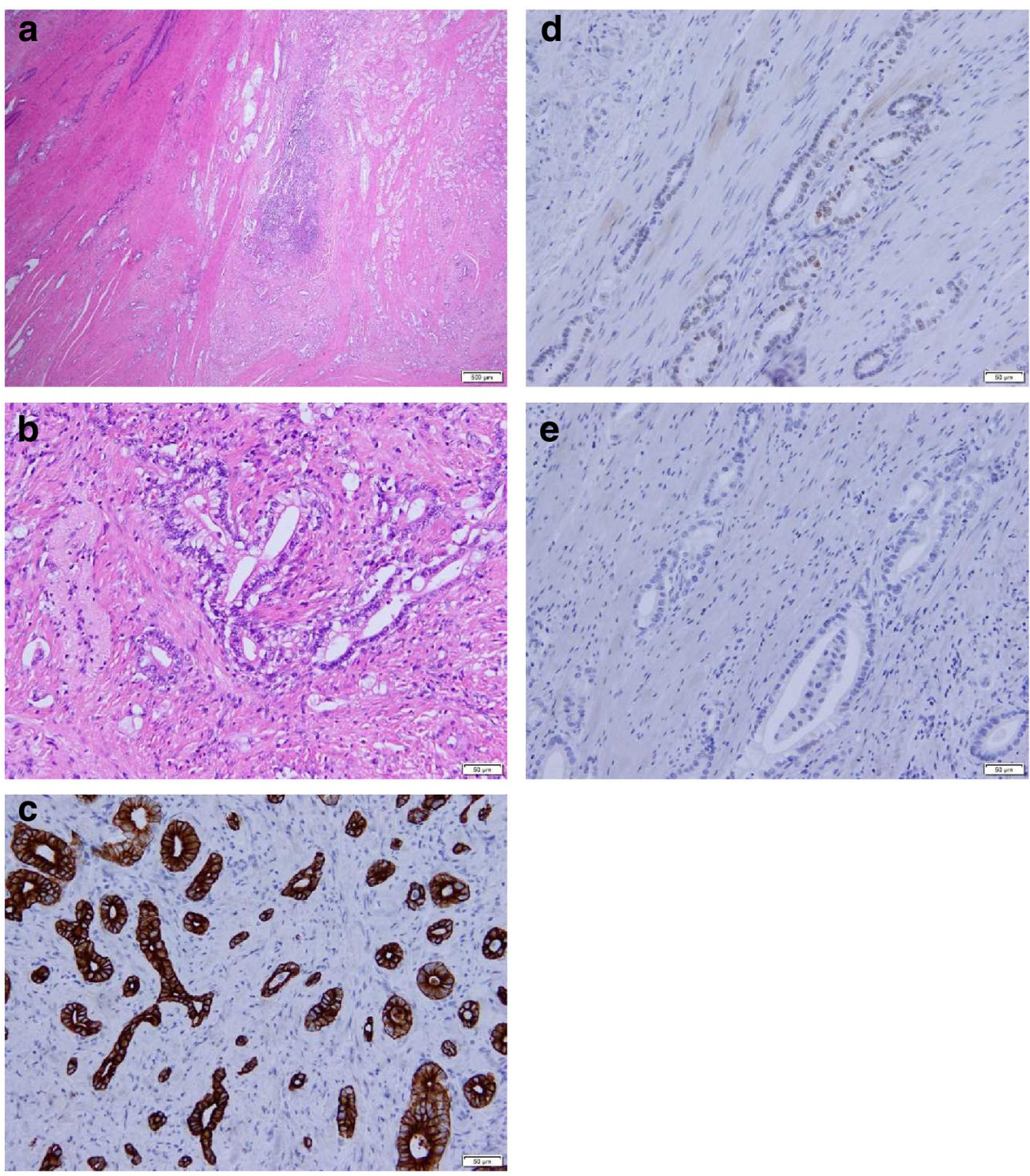

Fig. 2 Hematoxylin and eosin (H\&E) staining and immunohistochemistry of the resected specimen after pancreaticoduodenectomy. H\&E stains show well-differentiated to moderately differentiated adenocarcinoma (a, original magnification $\times 10$; $\mathbf{b}$, original magnification $\times 200$ ). Immunohistochemical stains show that the tumor cells were positive for cytokeratin 7 (c), original magnification $\times 200)$ and CDX-2 (d, original magnification $\times 200)$ and negative for cytokeratin $20(\mathbf{e}$, original magnification $\times 200)$ 

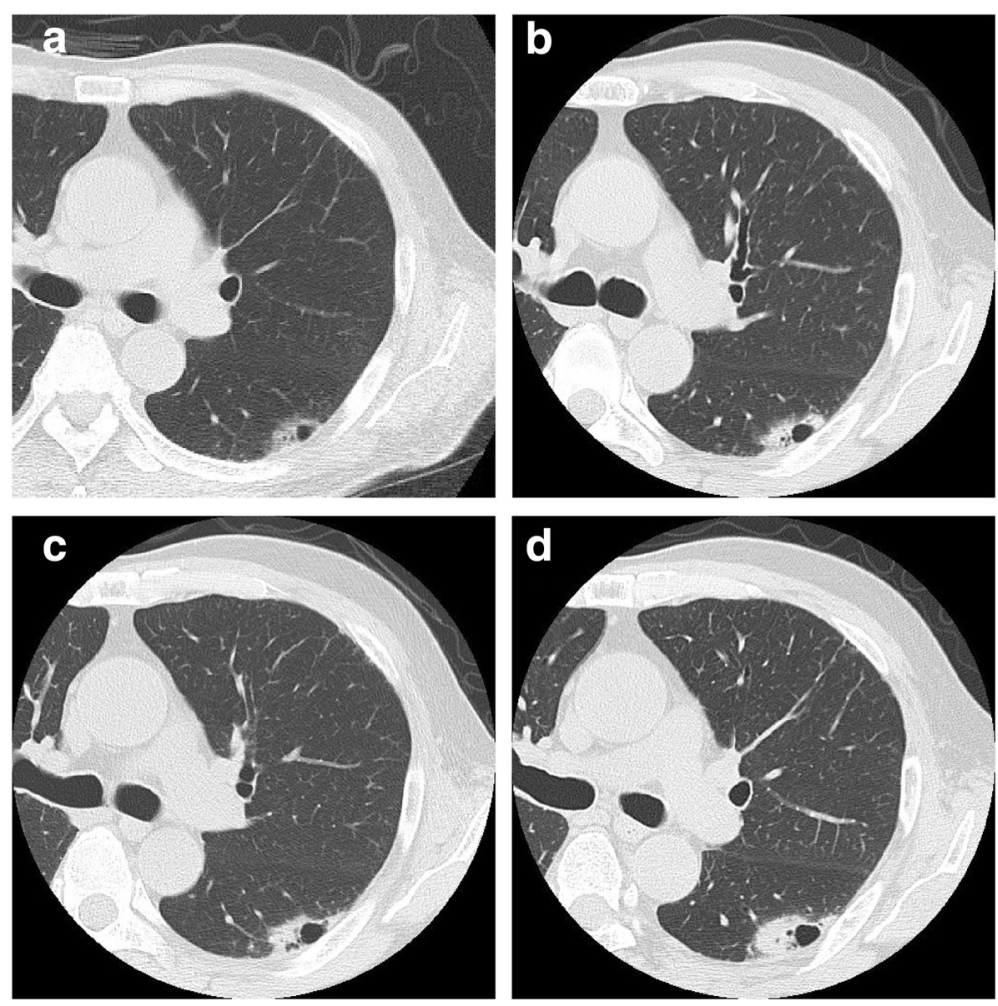

Fig. 3 Change in the air-space consolidation in the upper lobe of the left lung after pancreaticoduodenectomy: (a) 1 year, (b) 2 years, (c) 3 years, and $(\mathbf{d}) 3.5$ years

was a current smoker. His medical and family histories were unremarkable. He had undergone percutaneous transhepatic biliary drainage at a previous hospital because of obstructive jaundice without biliary tract stones. Abdominal computed tomography (CT) revealed thickening of the distal bile duct (Fig. 1a), and chest CT showed an air-space pattern in the upper lobe of the left lung (Fig. 1b). The patient's serum concentration of carcinoembryonic antigen was elevated at $15.1 \mathrm{ng} / \mathrm{ml}$, and that of carbohydrate antigen was within the reference range.

The patient underwent pancreaticoduodenectomy (PD) for the distal cholangiocarcinoma. Because of its small size, the lesion in the left lung could not be diagnosed accurately in terms of whether it was malignant and had the possibility of being pneumonia. Therefore, the lesion was observed. Histological examination of the resected specimen revealed well-differentiated adenocarcinoma invading the pancreas with five regional LN metastases (Fig. 2a, b). Immunohistochemical examination showed that the tumor cells were positive for cytokeratin 7 (CK7) (Fig. 2c) and CDX-2 (Fig. 2d) and negative for CK20 (Fig. 2e). According to the seventh edition of the TNM staging system of the Union for International
Cancer Control, the patient's disease was diagnosed as distal cholangiocarcinoma, T3N1M0, stage IIB. The patient received postoperative adjuvant chemotherapy with the oral fluoropyrimidine S-1 (120 mg/body) for 6 months. S-1 was given for 4 weeks, followed by a 2week rest. This administration of S-1 was repeated every 6 weeks for up to four cycles.

Follow-up chest CT was periodically performed about every 4 months during the first 5 years after the initial surgery. Follow-up chest CT demonstrated that the airspace pattern in the upper lobe of the left lung had gradually enlarged to $36.8 \mathrm{~mm}$ in diameter with no additional lesions for 3.5 years after PD (Fig. 3a-d). The patient was asymptomatic, and a physical examination including assessment of respiratory function revealed no remarkable findings. The patient's serum concentration of carcinoembryonic antigen was slightly elevated at 5 . $6 \mathrm{ng} / \mathrm{ml}$. Biochemical parameters except for carcinoembryonic antigen were within the reference ranges. Positron emission tomography with ${ }^{18} \mathrm{~F}$-fluorodeoxyglucose CT detected abnormal uptake in the left lung lesion with a maximum standardized uptake value of 4.30 (Fig. 4). A transbronchial lung biopsy showed malignant findings. However, the definitive diagnosis could not be established. 


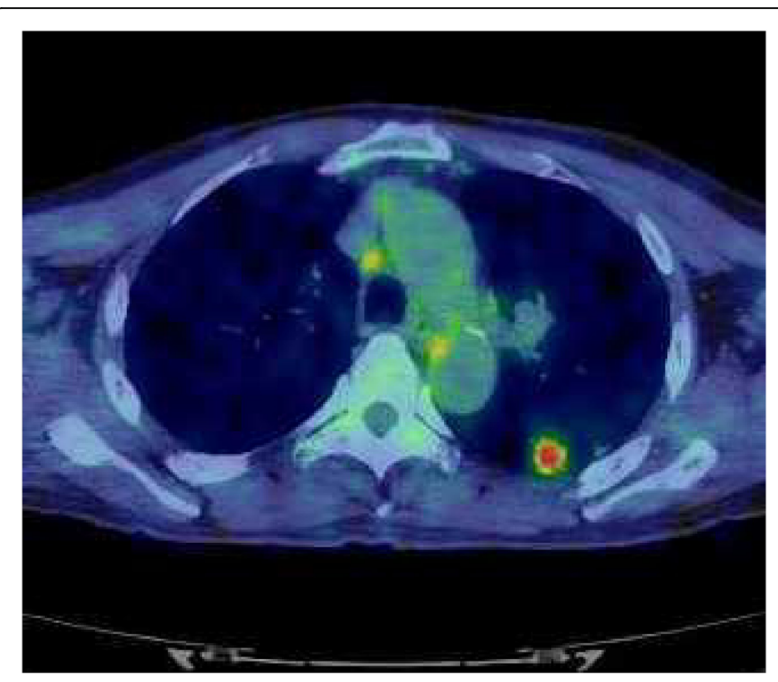

Fig. $4{ }^{18}$ F-fluorodeoxyglucose computed tomography revealing abnormal uptake in the lesion of the left lung with a maximum standardized uptake value of 4.30

With differential diagnoses of either primary lung cancer or solitary pulmonary metastasis from distal cholangiocarcinoma, thoracoscopic left S6 segmentectomy with LN dissection was performed. Histologically, the resected lung specimen showed adenocarcinoma tissue characterized by the formation of irregular tubular structures with lepidic growth (Fig. 5a, b). Immunohistochemical examination revealed that the tumor cells were positive for CK7 and CDX-2 (Fig. 5c, d), similar to the cholangiocarcinoma, and negative for thyroid transcription factor-1, napsin A, and CK20 (Fig. 5e-g). Additionally, an LN metastasis was detected in a resected regional $\mathrm{LN}$. On the basis of these findings, we finally diagnosed the patient with a solitary synchronous pulmonary metastasis from distal cholangiocarcinoma.

\section{Discussion}

To the best of our knowledge, this is the first report of resection of a solitary synchronous pulmonary metastasis with lepidic growth from distal cholangiocarcinoma 3.5 years after PD. This patient's course suggests two important clinical issues. First, surgical resection of a synchronous pulmonary metastasis from distal cholangiocarcinoma might offer better long-term survival than nonsurgical treatments. Although surgical resection is the only curative treatment for distal cholangiocarcinoma, more than half of patients who undergo curative resection develop recurrences [7], and the median survival time is $<12$ months $[1,2]$. The lung is the fourth most common site of recurrence after resection of cholangiocarcinoma [5]. In general, systemic chemotherapy
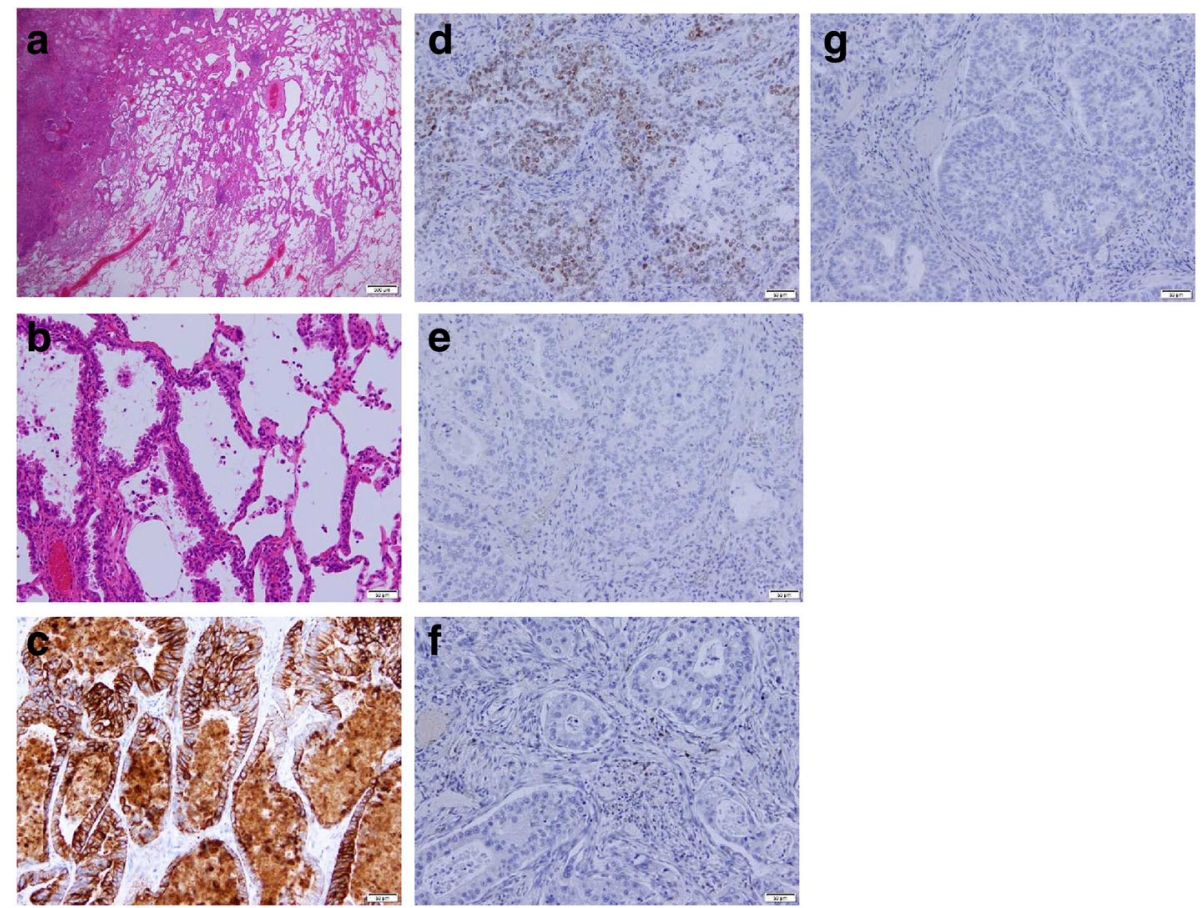

Fig. 5 The resected lung specimen resembled the previous distal cholangiocarcinoma. Histopathological examination (H\&E staining) of the lung tumor showed tumor cells forming irregular tubular structures with a lepidic pattern (a, original magnification $\times 10$; $\mathbf{b}$, original magnification $\times$ 200). Immunohistochemically, the tumor cells were positive for cytokeratin 7 (c), original magnification $\times 200)$ and CDX-2 (d, original magnification $\times 200$ ) and negative for thyroid transcription factor 1 (e, original magnification $\times 200)$, napsin A (f, original magnification $\times 200)$, and cytokeratin $20(\mathbf{g}$, original magnification $\times 200)$ 
or radiation therapy are considered for patients with recurrence, including recurrence of pulmonary metastases from cholangiocarcinoma. However, the clinical outcomes are not satisfactory [1, 2]. A few recent reports have described the effectiveness of resection for treatment of recurrent cholangiocarcinoma in select patients [3-6]. One study on metachronous pulmonary metastasectomy from cholangiocarcinoma revealed that surgery for pulmonary metastasis should be considered in patients with a longer initial disease-free interval [5]. In our patient, we surgically resected a slow-growing lesion in the upper lobe of the left lung with no additional lesions 3.5 years after the initial surgery because the definitive diagnosis could not be established (primary lung cancer versus solitary pulmonary metastasis from distal cholangiocarcinoma). The lesion in the left lung was histopathologically diagnosed as a solitary synchronous pulmonary metastasis. This case indicates that patients with synchronous pulmonary metastasis may have long survival and that pulmonary resection is possible in these patients.

Second, pulmonary metastasis from distal cholangiocarcinoma may exhibit lepidic growth, making it difficult to distinguish from primary lung cancer. In general, pulmonary metastasis is typically described as one or multiple nodules on radiological imaging. However, metastatic pulmonary adenocarcinoma rarely shows lepidic growth on radiological or histopathological examination $[8,9]$. Chest CT of pulmonary metastasis from the pancreas, colon, and stomach shows lepidic growth associated with airspace patterns, such as air bronchograms, ground-glass opacities, and consolidation [9]. Additionally, lepidic pulmonary metastasis from perihilar cholangiocarcinoma was reported in an autopsy case [10]. In our patient, preoperative chest CT before PD showed an air-space pattern in the upper lobe of the left lung. The lesion in the lung was diagnosed as pulmonary metastasis with a lepidic pattern from distal cholangiocarcinoma by immunohistochemical examination of the resected specimen 3.5 years after the initial surgery. A regional LN metastasis of the lung was also detected. Finally, we diagnosed the patient with a solitary synchronous pulmonary metastasis from distal cholangiocarcinoma.

\section{Conclusions}

We report the first case, to the best of our knowledge, of a slow-growing synchronous pulmonary metastasis with lepidic growth from distal cholangiocarcinoma resected 3.5 years after the initial surgery. This case is also extremely rare in that we were able to observe the course of the synchronous pulmonary metastasis for a long period of time. Surgical resection might offer better long-term survival to patients with synchronous pulmonary metastasis from distal cholangiocarcinoma than nonsurgical treatments. Pulmonary metastasis from distal cholangiocarcinoma may exhibit a lepidic pattern, making it difficult to distinguish from primary lung cancer. Therefore, pulmonary resection should be considered for patients with lepidic lesions who have been diagnosed with distal cholangiocarcinoma because of the possibility of synchronous pulmonary metastasis.

\section{Abbreviations \\ CK: Cytokeratin; CT: Computed tomography; LN: Lymph node; PD: Pancreaticoduodenectomy \\ Funding \\ The authors declare that they received no funding related to this case report. \\ Availability of data and materials \\ Data sharing is not applicable to this article, because no datasets were generated or analyzed during the current study. \\ Authors' contributions \\ TS wrote the manuscript. YF and SH supervised the writing of the manuscript. The other coauthors collected the data and discussed the content of the manuscript. All authors read and approved the final manuscript.}

\section{Ethics approval and consent to participate}

Not applicable.

\section{Consent for publication}

Written informed consent was obtained from the patient for publication of this case report and any accompanying images. A copy of the written consent is available for review by the Editor-in-Chief of this journal.

\section{Competing interests}

The authors declare that they have no competing interests.

\section{Publisher's Note}

Springer Nature remains neutral with regard to jurisdictional claims in published maps and institutional affiliations.

\section{Author details}

${ }^{1}$ Division of Surgical Oncology, Department of Surgery, Faculty of Medicine, Tottori University, 36-1 Nishi-cho, Yonago 683-8504, Japan. ${ }^{2}$ Division of Organ Pathology, Department of Pathology, Faculty of Medicine, Tottori University, Yonago 683-8503, Japan.

Received: 17 July 2017 Accepted: 28 March 2018

Published online: 22 May 2018

\section{References}

1. Valle J, Wasan $\mathrm{H}$, Palmer DH, Cunningham D, Anthoney A, Maraveyas A, et al. Cisplatin plus gemcitabine versus gemcitabine for biliary tract cancer. $\mathrm{N}$ Engl J Med. 2010;362:1273-81.

2. Okusaka T, Nakachi K, Fukutomi A, Mizuno N, Ohkawa S, Funakoshi A, et al. Gemcitabine alone or in combination with cisplatin in patients with biliary tract cancer: a comparative multicentre study in Japan. Br J Cancer. 2010; 103:469-74.

3. Takahashi Y, Ebata T, Yokoyama Y, Igami T, Sugawara G, Mizuno T, et al. Surgery for recurrent biliary tract cancer: a single-center experience with 74 consecutive resections. Ann Surg. 2015;262:121-9.

4. Noji T, Tsuchikawa T, Mizota T, Okamura K, Nakamura T, Tamoto E, et al. Surgery for recurrent biliary carcinoma: results for 27 recurrent cases. World J Surg Oncol. 2015;13:82.

5. Yamada M, Ebata T, Yokoyama Y, Igami T, Sugawara G, Mizuno T, et al. Pulmonary metastasis after resection of cholangiocarcinoma: incidence, resectability, and survival. World J Surg. 2017:41:1550-7.

6. Song SC, Heo JS, Choi DW, Choi SH, Kim WS, Kim MJ. Survival benefits of surgical resection in recurrent cholangiocarcinoma. J Korean Surg Soc. 2011; 81:187-94. 
7. Komaya K, Ebata T, Shirai K, Ohira S, Morofuji N, Akutagawa A, et al. Recurrence after resection with curative intent for distal cholangiocarcinoma. Br J Surg. 2017;104:426-33.

8. Rosenblatt MB, Lisa JR, Collier F. Primary and metastatic bronchiolo-alveolar carcinoma. Dis Chest. 1967;52:147-52.

9. Gaeta M, Volta S, Scribano E, Loria G, Vallone A, Pandolfo I. Air-space pattern in lung metastasis from adenocarcinoma of the Gl tract. J Comput Assist Tomogr. 1996;20:300-4.

10. Nagayoshi Y, Yamamoto K, Hashimoto S, Hisatomi K, Doi S, Nagashima S, et al. An autopsy case of lepidic pulmonary metastasis from cholangiocarcinoma. Intern Med. 2016;55:2849-53.

Submit your next manuscript to BioMed Central and we will help you at every step:

- We accept pre-submission inquiries

- Our selector tool helps you to find the most relevant journal

- We provide round the clock customer support

- Convenient online submission

- Thorough peer review

- Inclusion in PubMed and all major indexing services

- Maximum visibility for your research

Submit your manuscript at www.biomedcentral.com/submit
Biomed Central 